\title{
Restoration of Videos Degraded by Local Isoplanatism Effects in the Near-Infrared Domain
}

\author{
Magali Lemaitre*, Olivier Laligant*, Jacques Blanc-Talon ${ }^{+}$and Fabrice Mériaudeau* \\ * Le2i Laboratory, Université de Bourgogne, Le Creusot, France \\ + CEP, DGA, Arcueil, France
}

Received 16th May 2008; accepted 24th March 2009

\begin{abstract}
When observing a scene horizontally at a long distance in the near-infrared domain, degradations due to atmospheric turbulence often occur. In our previous work, we presented two hybrid methods to restore videos degraded by such local perturbations. These restoration algorithms take advantages of a space-time Wiener filter and a space-time regularization by the Laplacian operator. Wiener and Laplacian regularization results are mixed differently depending on the distance between the current pixel and the nearest edge point. It was shown that a gradation between Wiener and Laplacian areas improves results quality, so that only the algorithm using a gradation will be used in this article. In spite of a significant improvement in the obtained images quality, our restoration results greatly depend on the segmentation image used in the video processing. We then propose a method to select automatically the best segmentation image.
\end{abstract}

Keywords: atmospheric turbulence, local degradation, automatic segmentation, adaptive restoration.

\section{Introduction}

Atmospheric turbulence can severely degrade ground-to-ground acquisition images and videos in the case of important temperature fluctuations. We address here the case of local atmospheric perturbations, particularly in a military context for distant surveillance of dangerous areas in the near-infrared domain.

Turbulence strength essentially depends on climatic conditions and on the distance between the scene and the camera. The video sequence we tested our algorithm on has been provided by DRDC Valcartier, Canada, and it was acquired during the NATO RTG40 campaign in New Mexico in 2005. In our sequence acquisition conditions (horizontal observation in the troposphere, at a distance of about $1 \mathrm{~km}$ ), atmospheric perturbation can be efficiently simulated by local blurring and warping and possibly additive noise. Each frame can then be split into mostly regular areas degraded by the same perturbation (local isoplanatism).

Several authors developped techniques to restore videos degraded by atmospheric turbulence during groundto-ground acquisition. Frakes et al. propose to detect an atmospheric vector field and to use it in their distortion compensation process [1]. Yaroslavsky et al. first process and then fuse visible and thermal sequences to obtain better results [2], or first use a median filter to obtain an unwarped image and then detect moving objects [3]. Kopeika et al. compute atmospheric effects with different modulation transfer functions (MTFs) described by

Correspondence to: < fabrice.meriaudeau@u-bourgogne.fr >

Recommended for acceptance by David Fofi

ELCVIA ISSN:1577-5097

Published by Computer Vision Center / Universitat Autonoma de Barcelona 
analytical functions, and restore their images by a simple Wiener filter [4]. Fraser and Lambert detail a method to detect a local atmospheric point-spread function (PSF) through a region-of-interest Wiener filter, and they compare their new registration and restoration method with windowed cross-correlation previously used [5].

In our last article [6], we proposed two hybrid restoration algorithms based on a mixing of two space-time processings: a Wiener filter and a regularization by the Laplacian operator. Wiener and Laplacian regularization results are mixed differently depending on the distance between the current pixel and the nearest edge point. A gradation between Wiener and Laplacian areas improves results quality, then only the algorithm using a gradation will be used in this article. A significant improvement has been shown in the obtained images quality, but our restoration results greatly depend on the segmentation image used in the video processing. We then propose a method to select automatically the best segmentation image.

First we recall what local isoplanatism is. Then we explain the general algorithm used to process locally a video sequence, we show some restoration results and we analyse them. Therefore the segmentation process is detailed, and restoration results are given and compared with previous ones. Finally, a conclusion and some perspectives are given.

\section{Local Isoplanatism Theory}

\subsection{Definition}

Atmospheric turbulence induce varying perturbations on optical beams, according to beams propagation directions. On Fig. 1 is given an example where two beams coming from the same object cross a thin turbulent layer.

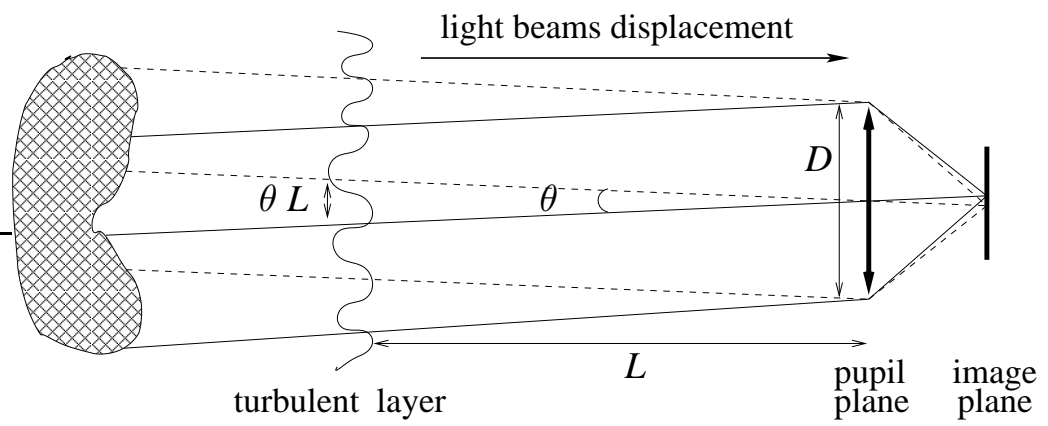

Figure 1: Origin of different atmospheric perturbations $(\theta$ is the angle between the two beams, $L$ is the distance between the turbulent layer and the pupil, and $D$ is the pupil diameter).

Three degradation types can occur:

- Anisoplanatism: If $|\theta L|>D$, the turbulent layer areas met by the two beams have no common part. The beams are perturbed by two completely different degradations.

- Local isoplanatism: If the observed object has sufficiently small angular dimensions $\theta$, beams originating from any point on the object and arriving on the pupil can be considered to have encountered almost identical regions of the perturbing layer [7]. That will be translated on the related image by areas where the perturbation is the same.

- Total isoplanatism: When $\theta \approx 0$, the two beams suffer from exactly the same perturbation.

\subsection{Isoplanatic angle}

In the case of horizontal propagation on a distance $L$ in a turbulent media, the structure constant $C_{N}^{2}$ which corresponds to turbulence influence on optical propagation, can be approximated by a constant and the isoplanatic 
angle $\theta_{0}$ is then defined by [8]:

$$
\theta_{0}=0.95\left(\frac{2 \pi}{\lambda}\right)^{-6 / 5} L^{-8 / 5}\left(C_{N}^{2}\right)^{-3 / 5} .
$$

Knowing that $\lambda=0.86 \mu m, L=1000 m$ and $C_{N}^{2} \approx 1.10^{-12} m^{-2 / 3}$, we obtain an estimated value for the studied video sequence: $\theta_{0} \approx 1.38 \mu \mathrm{rd}$.

\subsection{Instantaneous Field Of View (IFOV)}

The IFOV is the maximal angle covered by a single detecting element in the image plane. It can be estimated by using simple trigonometry. The vertical IFOV is then approximated by $\frac{H}{N L}$ where $H$ is the observed object real height, $N$ is the vertical pixel number needed to represent $H$ on the detecting matrix, and $L$ is the target-sensor distance.

Knowing the observed object real height $(1.5 \mathrm{~m})$ and having estimated the related pixel number $N$ (107 pixels), we then obtain an estimated vertical IFOV value for the studied video sequence: $I F O V \approx 14.0 \mu \mathrm{rd}$.

Comparing $\theta_{0}$ and IFOV values, we conclude that IFOV covers a larger angle than $\theta_{0}$ so that our sequence should be degraded by anisoplanatism. But during the acquisition, the exposure time was about $33 \mathrm{~ms}$ which is not weak enough to "freeze" the turbulence effects on each frame. These ones can then be considered as averaging of several short exposure images, and that is why they are composed of different areas with the same atmospheric perturbation on average. They finally fit with local isoplanatism assumptions.

\section{Video sequence processing algorithm and results analysis}

\subsection{Two spatial and temporal methods}

- A space-time Wiener filter :

The D. Fraser's and A. Lambert's algorithm [5] schemed on Fig. 2 has been used to process our video sequence. Its principle is to detect a local space-varying point-spread function (PSF) describing the atmospheric turbulence. The PSF is found by using a Wiener filter acting on regions-of-interest of a reference image and each frame of the sequence. The reference image is initially the sequence average, and is updated after each deconvolution pass of the complete sequence. The process is repeated until the absolute difference between the two last average images is minimized. In practice, one or two deconvolutions of the complete sequence are sufficient.

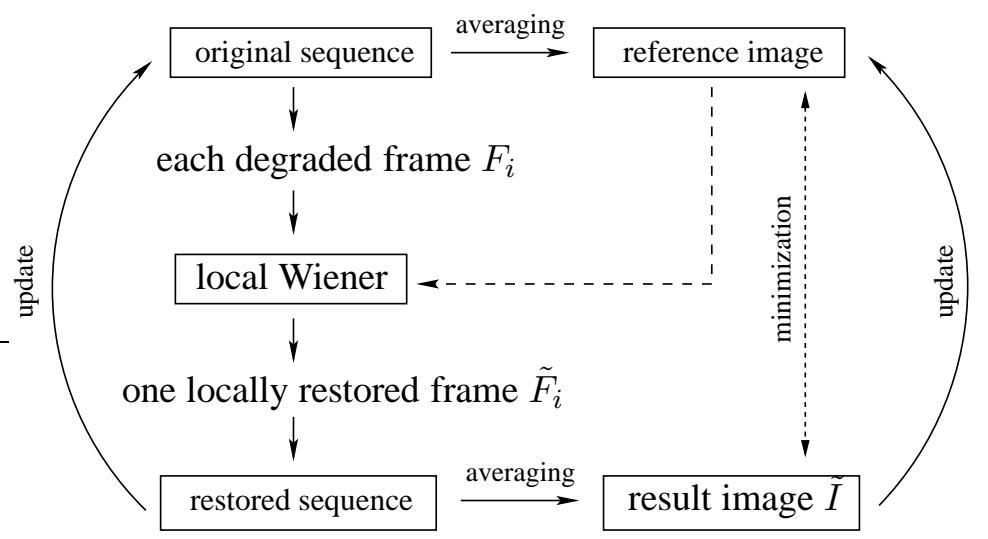

Figure 2: Sequence processing by a local and temporal Wiener filter.

- A space-time Laplacian regularization :

The same general scheme has been used, replacing the local Wiener filter step by a local regularization by the Laplacian operator. 


\subsection{Spatial and temporal methods results and analysis}

On Fig. 3 are shown our first restoration results. The processed sequence is compound of 100 frames of 256 x 256 pixels size from an original sequence provided by DRDC Valcartier. It was acquired during night and the objects were lighted by a laser. We consider that possible speckle noise is eliminated with spatial integration due to the large target-sensor distance.

Looking at Fig. 3, we can observe that averaging allows to strongly decrease noise in the reference images but the local Laplacian regularization allows to improve noise removal. Also we can choose the best suited parameter of the local Wiener filter in order to remove the maximum of the remaining blur so as to obtain clearer edges.

We made our processing on MATLAB. Local restoration computing time is from few minutes to about one hour depending essentially on the frame number in the processed sequence, on their size and on the number and size of regions-of-interest (ROI) used to process each frame. 32 × 32 pixels ROI were used to obtain results shown on Fig. 3.

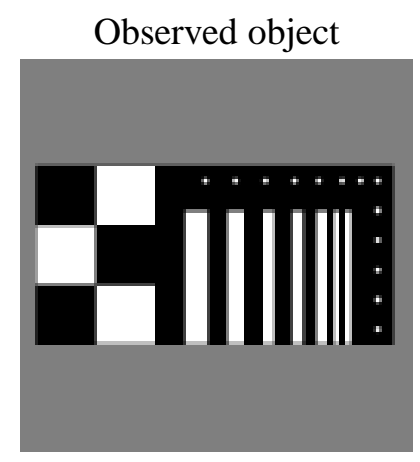

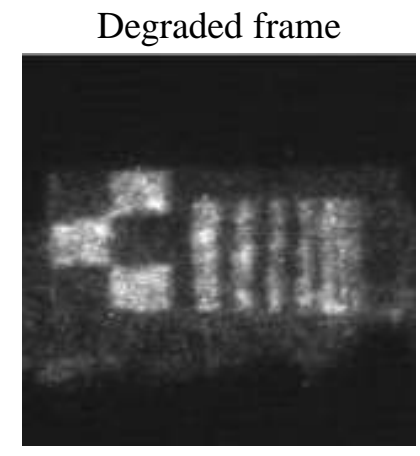

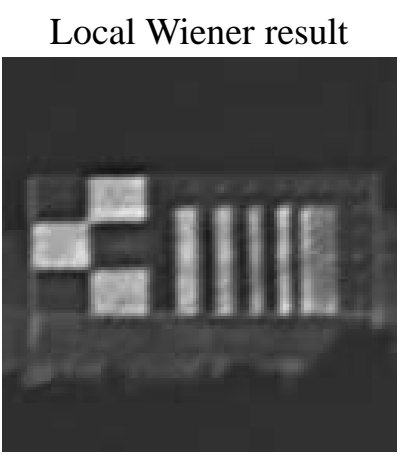

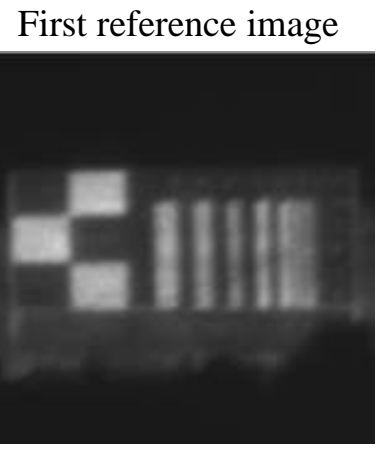

Local Laplacian result

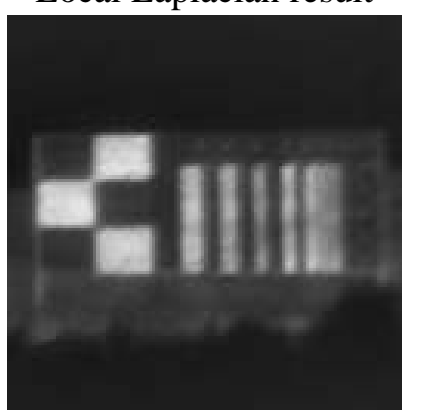

Figure 3: First video sequence restoration results.

Several criteria have been used to compare and appreciate our restoration results. We first calculated the mean variances in the three white squares and also in the three black squares on the checkerwork: the results are noted on Tab. 1. The local Laplacian regularization gives the better result.

Table 1: Mean variance values in black and white squares for images resulting from the processed sequence (Contrast estimation by $\frac{\text { Max-Min }}{\text { Max }+ \text { Min }}$ is given for a better comparison).

\begin{tabular}{|l|c|c|c|}
\hline Image & Black squares variance & White squares variance & Contrast \\
\hline Degraded frame & 102.1 & 540.9 & 0.89 \\
\hline First reference image & 11.6 & 84.1 & 0.82 \\
\hline Local Laplacian result & 11.1 & 64.0 & 0.80 \\
\hline Local Wiener result & 16.4 & 98.2 & 0.91 \\
\hline
\end{tabular}


The other criteria concern edges areas. We compared the mean slope of horizontal and vertical transitions between black and white squares: the local Wiener filter gives the steepest mean slope. We also computed the modulation transfert function (MTF) of each mean transition between black and white squares, which provides a quantified and graphic representation of simultaneous qualities of contrast and clearness. The mean transition MTF is the modulus of the Fourier transform of its derivative, and is then normalized to range between 0 and 1. According to Fig. 4(a), the local Wiener filter gives the best MTF. Furthermore for each result, we compared the correlations of each mean transition with the ideal one. According to Fig. 4(b), the local Laplacian regularization gives a slightly better result than the local Wiener filter, but this is due to the fact that the Wiener result is more constrasted than the Laplacien one and that creates oscillation before and after the edge.

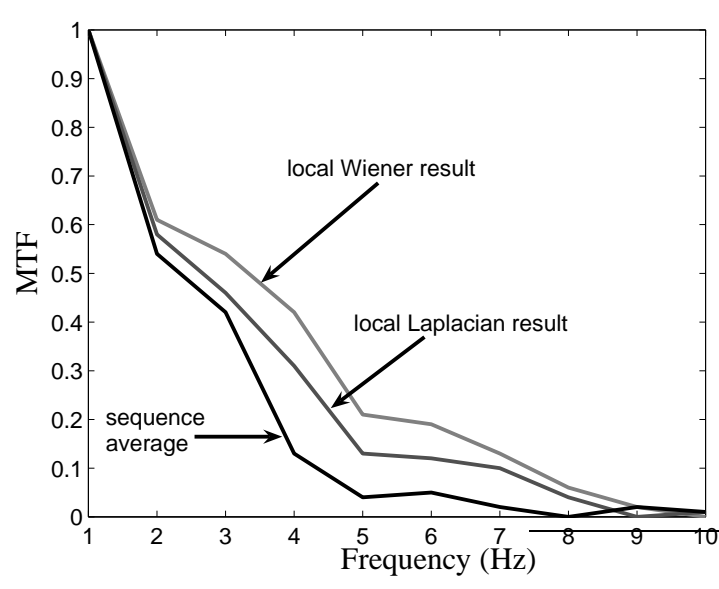

(a)

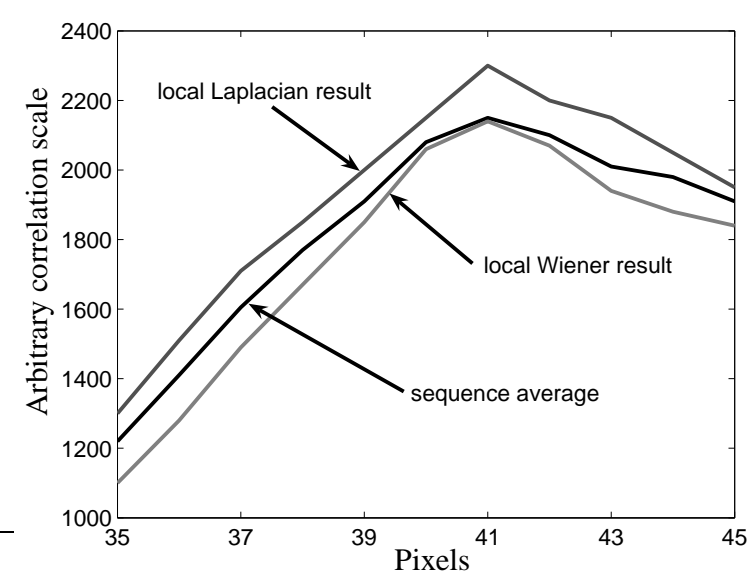

(b)

Figure 4: First restoration results analysis. (a) Mean transitions MTFs for the reference image, the local Wiener result and the local Laplacian result. (b) Correlation peaks of the same images mean transitions with the ideal one.

To summarize, the local Laplacian regularization allows to improve noise removal on uniform areas whereas the local Wiener filter allows to get rid of a large part of the remaining blur on edges. A hybrid method which takes advantages of these two methods is thereafter proposed.

\subsection{Mixing algorithm using a gradation}

This algorithm mixes the space-time methods results according to each pixel membership of an edge area or a uniform one. Using a gradation between edge and uniform areas improves results quality [6]. This algorithm is compound of two steps: a segmentation step and a fusion one.

\subsubsection{Segmentation image}

We need a segmentation image (i.e. an edge image) to determine later areas where the Wiener result will be kept, areas where the Laplacian result will be kept, and areas where both results will be fused. It is obtained with the Canny-Deriche filter. To limit false edge detection, we use the three images we have in input: the reference image, the local Wiener result and the local Laplacian result. On the final segmentation image, an edge point is kept only if it's present on at least two of the three used segmentation images.

In a first time, two segmentation thresholds $(t h r)$ have been arbitrarily chosen: the first one allows to detect small white circles above and on the right of vertical bars (Fig. 5(b)), while the second one allows to obtain a "clean" segmentation, i.e. without parasite edge (Fig. 5(c)). Automatic selection of segmentation threshold is under investigation, with a method based on detected edge points number study. 


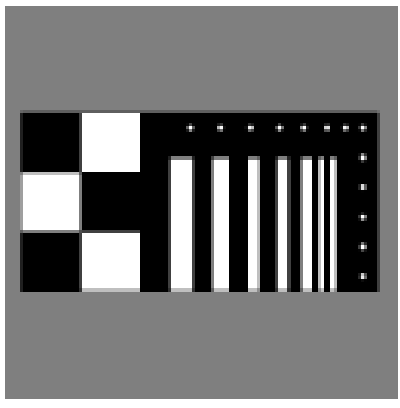

(a)

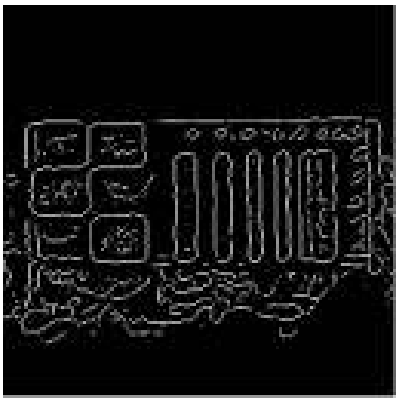

(b)

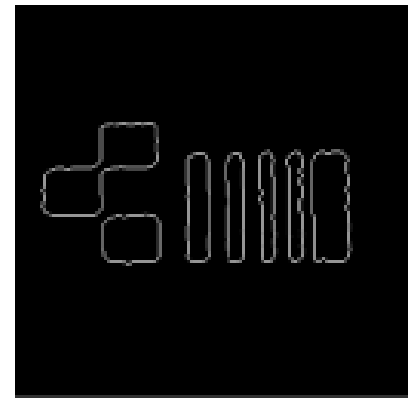

(c)

Figure 5: Observed object $(a)$ and the 2 used segmentation images: $(b)$ thr $=0.04$ and $(c)$ thr $=0.35$.

\subsubsection{Fusion}

In the fusion step, the restoration result is adapted according to the distance between the current pixel and the nearest edge point: the local Wiener result will be dominant near edges whereas in uniform areas the local Laplacian result will be better adapted. Between these two cases, some pixels can be considered as belonging to both edge area and uniform area. A gradation is then used to pass from the Wiener result to the Laplacian result, which allows simultaneous attenuation of the small gray level difference between them. A varying weighting coefficient $\alpha$ is added depending on the proximity of the current pixel to the nearest edge point. For each pixel, the following formula is used:

$$
\forall i, j, W L M(i, j)=\alpha(c) L W R(i, j)+(1-\alpha(c)) L L R(i, j),
$$

where WLM is the Wiener and Laplacian Mixing result, LWR is the local Wiener result, LLR is the local Laplacian result, and $c$ is the distance transform of the segmentation image (i.e. the distance card obtained from the segmentation image and representing the distance between each pixel and the nearest edge point). The coefficient $\alpha$ is such as $0 \leq \alpha(c) \leq 1$.

The closer to an edge point, the higher $\alpha$, and conversely. The gradation is made on several pixels from the center of the mean transition on the local Wiener result, according to the pixels number $(N)$ needed for this mean transition (Figs. 6(a) and 6(b)). $N$ is computed for each edge in the image. In the case where it is even, the two middle pixels are considered as central pixels. WLM results are shown on Figs. 7(a) and 7(b).

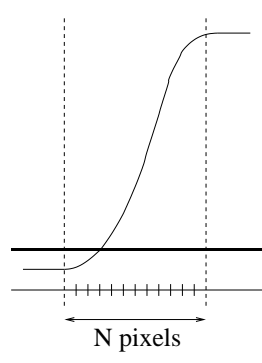

(a)

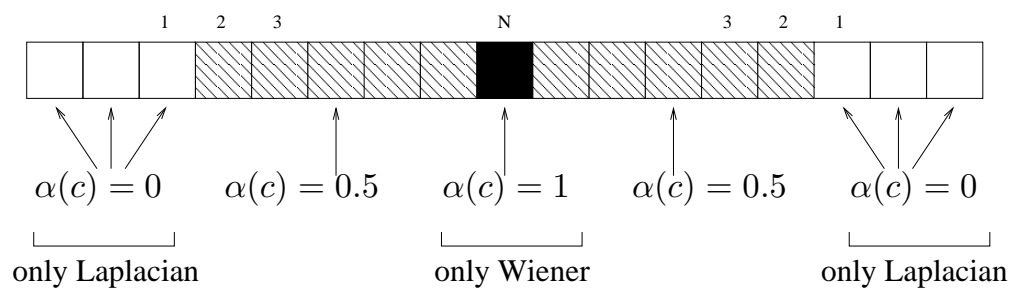

(b)

Figure 6: Transition example $(a)$ and processing areas determination with an odd transition pixel number $(b)$.

\subsubsection{Results analysis}

Analysis of our mixing results have been realized with the same criteria than those previously used, and similar results to previous ones have been found (Fig. 8): WLM results mean transitions provide MTFs almost as good 


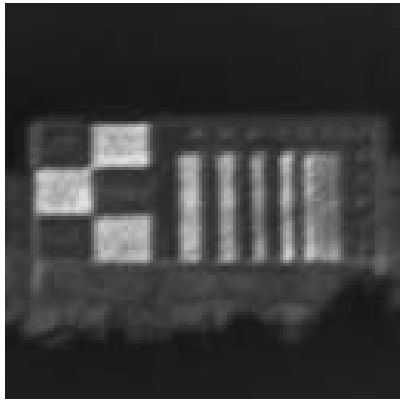

(a)

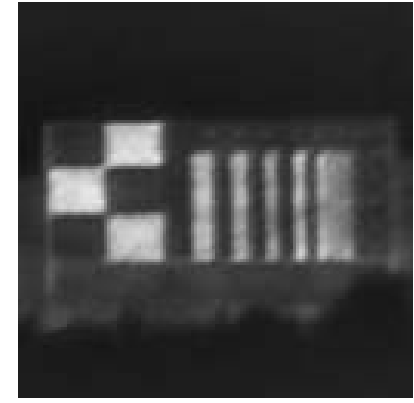

(b)

Figure 7: WLM results with $t h r=0.04(a)$ and $t h r=0.35(b)$.

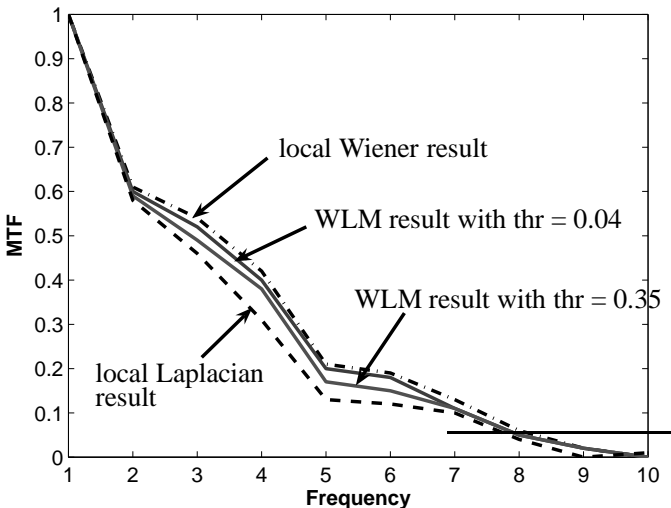

(a)

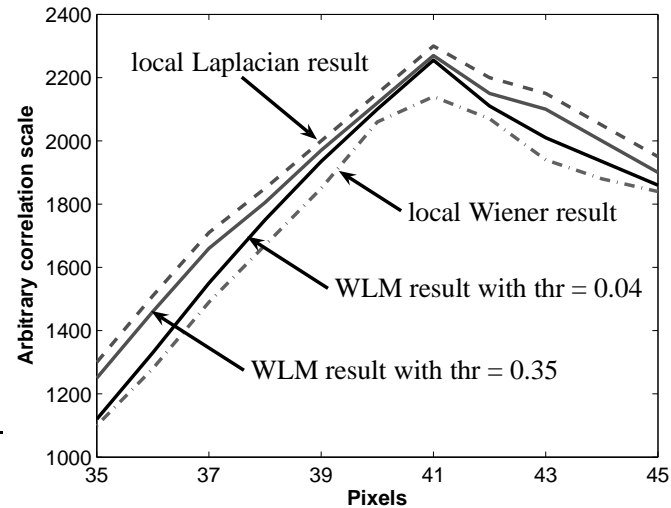

(b)

Figure 8: WLM restoration results analysis. (a) Mean transitions MTFs. (b) Correlation peaks with the ideal transition. WLM result with a low threshold provides at once a good MTF and a good correlation peak.

as those obtained with the local Wiener result, and correlation peak with the ideal transition has been improved compared with the local Wiener result. Moreover the Canny-Deriche filter has been tried on these results (Fig. 9), which allows us to conclude that white circles are better detected with a low threshold. Horizontal cuts have also been realized along small white circles above the vertical bars to determine the brought improvement concerning circles detection (Fig. 10). Results strongly depend on the chosen segmentation threshold: if the circles are not detected on the used segmentation image, the Laplacian result is applied and edges are smoothed.
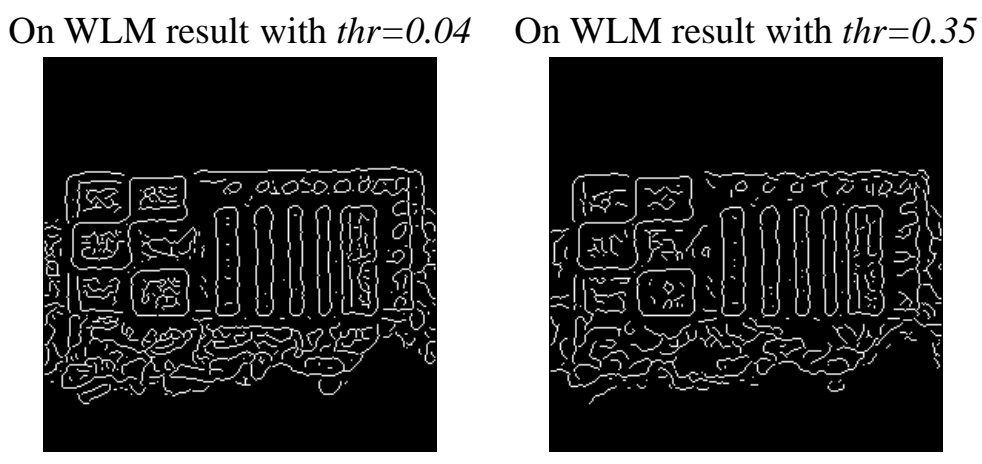

Figure 9: Canny-Deriche segmentations on WLM results. (Circles are better detectd with a low threshold.) 

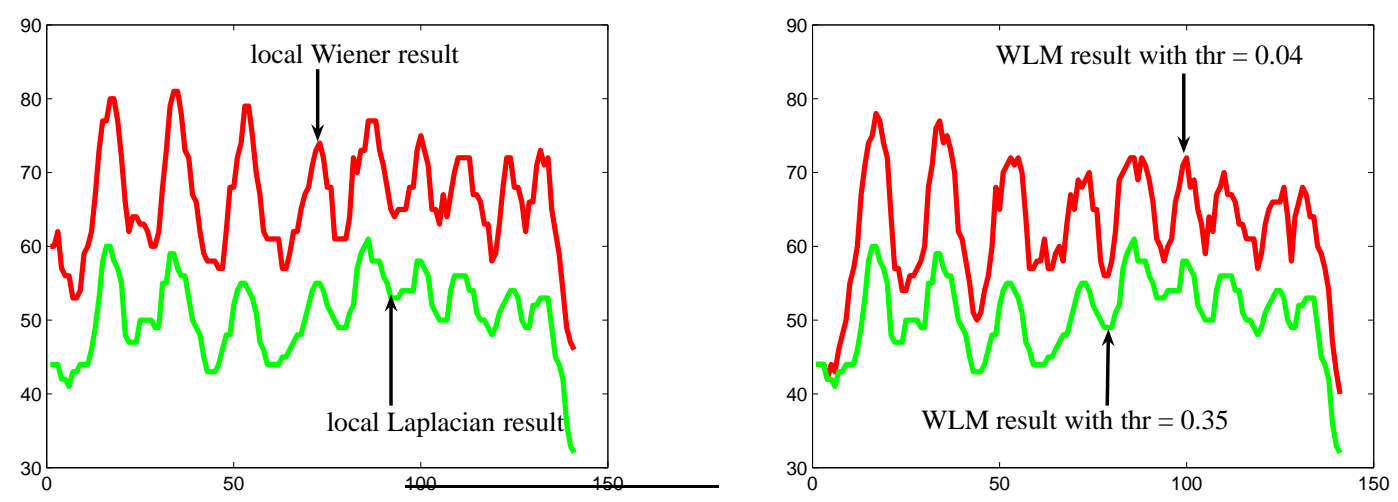

Figure 10: Horizontal cuts along white circles. For each curve, high gray levels represent detected white circles. On WLM results, circles are better detected with a low threshold.

\section{Automatic selection of the best segmentation image}

This section is dedicated to the improvement of the segmentation step. Restoration results strongly depend on the segmentation threshold. A too high threshold does not allow to detect smallest details in the scene, whereas with a too low threshold noise and textures appear. A method is proposed to find the best adapted segmentation threshold, then the new restoration result is analyzed.

\subsection{Ideal threshold determination}

A range of thresholds are applied to the three Canny-Deriche results (on the average image, on the local Wiener result and on the local Laplacian result). Each curve of the detected edge point percentages in function of thresholds is L-shaped. A small threshold results in too many false edges, where a large threshold results in strong edges only. We look for the best compromise between these two extreme cases. The most interesting segmentation images are those with points on the L-curve nearest to the origin. We choose to compute the threshold $t h r$ automatically by interpolation in order to minimize the Euclidean distance between the L-curve and the origin (Fig. 11) (the thresholds around $t h r$ don't let to bring additional information). The corresponding segmentation image is shown on Fig. 12(a).

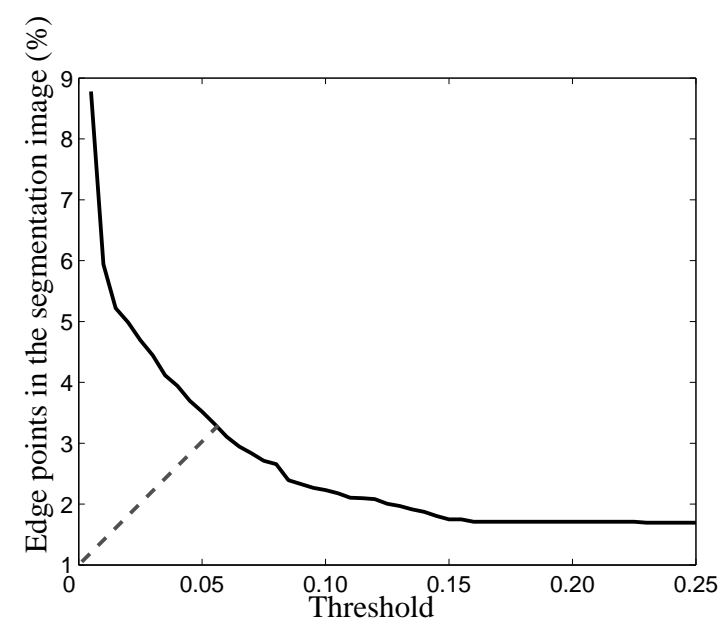

Figure 11: Automatic choice of the segmentation threshold for the average image. 


\subsection{New restoration result analysis}

The new restoration result of the WLM algorithm on the degraded video sequence is shown on Fig. 12(b). It visually seems less noisy than the result obtained with a low threshold (Fig. 7(a)) and white circles are clearer than on the result obtained with a high threshold (Fig. 7(b)).

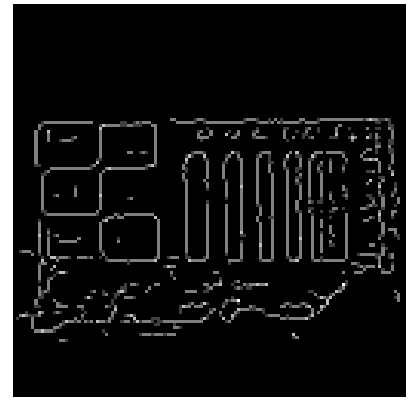

(a)

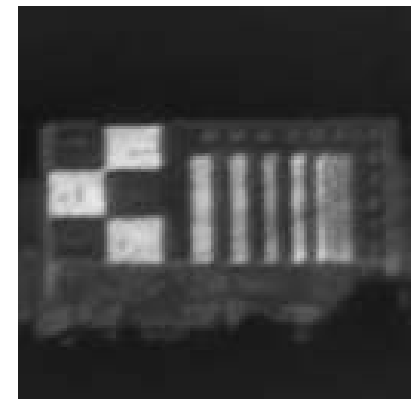

(b)

Figure 12: (a) Best segmentation image. (b) New WLM result.

The same criteria have been used to analyze this new restoration result and they confirm the quality of this result compared with the previous ones. Variances are weak in uniform areas (Tab. 2). Concerning mean transitions between two uniform areas, MTFs and correlation curves are very close to those found when using a low threshold $(t h r=0.04)$.

Table 2: Mean variance values in black and white squares for images resulting from the processed sequence.

\begin{tabular}{|l|c|c|}
\hline Image & Black squares variance & White squares variance \\
\hline Low threshold result & 14.9 & 88.2 \\
\hline High threshold result & 11.9 & 64.5 \\
\hline Ideal threshold result & 12.2 & 65.3 \\
\hline
\end{tabular}

Finally the Canny-Deriche filter has been applied on this new result (Fig. 13). Compared with Canny results on Fig. 9, textures are less detected than with a low threshold and white circles are better detected than with a high threshold. The only drawback is a certain noise presence due to textures.

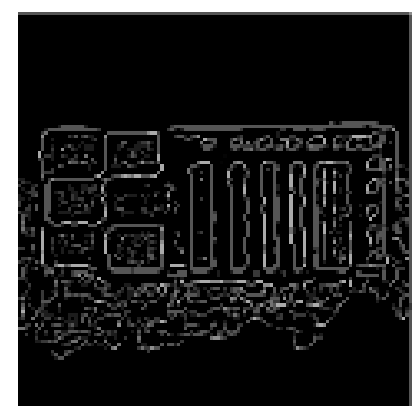

Figure 13: Canny-Deriche segmentation on the new WLM result. 


\section{Conclusion and perspectives}

An adaptive Wiener and Laplacian algorithm has been proposed to restore video sequences degraded by local perturbations due to atmospheric turbulence. Results strongly depend on the segmentation image found by the Canny-Deriche filter. The segmentation step has been improved to choose automatically the segmentation threshold. The restoration result is then the best compromise between details detection and avoidance of false edges. It satisfies simultaneously clarity criteria around edges and noise removal in uniform areas, which gives in practice an image adapted for both visualization and post-processing. Patterns are less noisy and more easily detected.

Further improvements could be added in the segmentation step, either for instance by processing textures during edge closing [9], or by separating textures before segmentation [10, 11].

\section{References}

[1] D.H. Frakes, J.W. Monaco, M.J.T. Smith, "Suppression of Atmospheric Turbulence in Video Using an Adaptive Control Grid Interpolation Approach", Proceedings of IEEE International Conference on Acoustics, Speech, and Signal Processing (ICASSP), USA, 1881-1884, 2001.

[2] L.P. Yaroslavsky, B. Fishbain, A. Shteinman, S. Gepshtein, "Processing and Fusion of Thermal and Video Sequences for Terrestrial Long Range Observation Systems", Proceedings of the 7th International Conference on Information Fusion, Sweden, 2:848-855, 2004.

[3] S. Gepshtein, A. Shteinman, B. Fishbain, L.P. Yaroslavsky, "Restoration of Atmospheric Turbulent Video Containing Real Motion Using Rank Filtering and Elastic Image Registration", Proceedings of the 12th European Signal Processing Conference (EUSIPCO), Austria, 477-480, 2004.

[4] Y. Yitzhaky, I. Dror, N.S. Kopeika, "Restoration of Atmospherically Blurred Images Using WeatherPredicted Atmospheric Modulation Transfer Function (MFT)", Proceedings of SPIE, Image Propagation Through the Atmosphere, 2828:386-396, 1996.

[5] D. Fraser, A. Lambert, "Information Retrieval from a Position-Varying Point Spread Function", Proceedings of Conference on Advanced Concepts for Intelligent Vision Systems (ACIVS), Belgium, 2004.

[6] M. Lemaitre, O. Laligant, J. Blanc-Talon, F. Mériaudeau, "Local Isoplanatism Effects Removal on Infrared Sequences", Proceedings of SPIE, Quality Control by Artificial Vision (QCAV) Conference, France, Vol. 6356, 2007.

[7] F. Roddier, "Effects of Atmospheric Turbulence in Optical Astronomy", Progress in Optics, 19:281376,1981 .

[8] D.L. Fried, "Anisoplanatism in Adaptive Optics", Journal of the Optical Society of America, 72(1):52-61, 1982.

[9] S. Philipp, P. Zamperoni, "Segmentation and Contour Closing of Textured and Non-Textured Images Using Distances between Textures", Proceedings of International Conference on Image Processing (ICIP), 3:125128, 1996.

[10] J. Gilles, "Décomposition et Détection de Structures Géométriques en Imagerie", PhD Thesis, ENS Cachan, 2006.

[11] J.-F. Aujol, G. Gilboa, S. Osher, "Structure-Texture Image Decomposition - Modeling, Algorithms, and Parameter Selection", International Journal of Computer Vision, 67(1):111-136, 2006. 